\title{
Adaptive Aperiodically Intermittent Synchronization for Complex Dynamical Network with Unknown Time-Varying Outer Coupling Strengths
}

\author{
Lihong Yan $\mathbb{D}^{1,2}$ and Junmin $\mathrm{Li}^{1}$ \\ ${ }^{1}$ School of Mathematics and Statistics, Xidian University, Xian 710071, China \\ ${ }^{2}$ College of Mathematics and Information Science, Xianyang Normal University, Xianyang 712000, China \\ Correspondence should be addressed to Lihong Yan; 092212@163.com
}

Received 3 April 2019; Accepted 15 May 2019; Published 29 May 2019

Academic Editor: Jean Jacques Loiseau

Copyright (C) 2019 Lihong Yan and Junmin Li. This is an open access article distributed under the Creative Commons Attribution License, which permits unrestricted use, distribution, and reproduction in any medium, provided the original work is properly cited.

In this paper, exponential synchronization problem of complex dynamical networks with unknown periodically coupling strengths was investigated. An aperiodically intermittent control synchronization strategy is proposed. Based on Lyapunov exponential stability theory, inequality techniques, and adaptive learning laws design, some sufficient exponential synchronization criteria for complex dynamical network with unknown periodical coupling weights are obtained. The numerical simulation example is presented to illustrate the feasibility of theoretical results.

\section{Introduction}

Complex dynamical networks, such as biological network, urban transportation network and human relationship network, are ubiquitous in natural world and social society generally. Researchers were mainly concerned with the issues of modelling, properties analysis, dynamical evolution, and synchronization control of complex dynamical networks. Among these issues involved, synchronization is one of the most interesting topics and has been extensively investigated [1-12]. There are mainly two kinds of strategies for synchronization of complex dynamical networks: one is to improve the network synchronization capability by changing the properties of the network itself, such as topology structure and coupling strengths. The other is to act on the network with external control injection, which is a representative of control theory, mainly including variable feedback control method, pinning control method [2,4], adaptive control method [5], event-triggered control [8], impulse control method, intermittent pinning control [9], and slide mode control method $[10,11]$.

We can see that most of the above control methods are continuous, which requires continuous information exchange and increases the cost of control. However, the discontinuous control method such as intermittent control, exerting intermittent control over the controlled objects, can reduce the amount of the transmitted information and be much more economic. Zochowski firstly introduced intermittent control into dynamic control systems [12]. After that, many researchers pay attention to this control strategy and many large-scale dynamical systems are controlled successfully with the help of intermittent control. So, it can also be applied to realize synchronization of complex dynamical networks [13-23].

Over the first few years, the intermittent controller which can help realizing synchronization for complex dynamical networks applied is usually periodical. In papers [13-16], the authors investigated finite-time synchronization of complex dynamical networks with multilink, time delay, and multiswitch periods using periodically intermittent control scheme. Zhao and Cai [17] investigated the exponential synchronization of complex delayed dynamical networks with uncertain parameters adopting the intermittent control scheme, basing on the Lyapunov stability theory combined with the method of the adaptive control. Then semiperiodically intermittent control method was proposed, by 
applying multiple Lyapunov function method and the modedependent average dwell time approach. Qiu [18] derived less conservative synchronization criteria and the synchronization problem for switched complex networks with delayed coupling via semiperiodically intermittent control technique and a mode-dependent average dwell time method was studied. Liu [19] addressed synchronization problems of timedelay coupled network by aperiodically intermittent control. In 2017, with the help of aperiodically intermittent control method, Liu [20] discussed finite-time synchronization of delayed dynamical networks and showed the convergence time does not depend on control widths or rest widths. Combining intermittent control with pinning control scheme, which can greatly reduce control cost, researchers presented some conclusions about intermittent pinning control. Wang [21] proposed a new differential inequality, dealing with the synchronization problem of complex dynamical network with constant coupling, discrete-delay coupling, and distributed-delay coupling. The synchronization problem of switched complex networks with unstable modes and stochastic complex-valued dynamical networks by aperiodically intermittent adaptive control are discussed in $[22,23]$, respectively.

It is noteworthy that the above aperiodically intermittent adaptive control results can ensure all nodes of complex dynamical network realizing exponential synchronization, which can make the system tend to equilibrium state much more quickly compared with the asymptotic synchronization of networks [24]. In [25], the exponential synchronization problem of a class of hybrid coupled complex dynamical network with time-varying delay is studied. By designing appropriate intermittent feedback controller, a new synchronization criterion is presented. In paper [26], a kind of complex network model with directional topology and timedelay is studied, and sufficient conditions for global exponential synchronization of the system are obtained. Zhang et al. [27] investigated exponential stability of stochastic differential equations with impulse effects. As seen from the above literatures, exponential synchronization of complex dynamical network via aperiodically intermittent control could save the control cost, which is significant in practical applications.

What is more, with the research of complex dynamic network control, researchers found that the differences of nodes and coupling modes play an important role for the evolution of complex dynamical networks. In addition, many practical complex dynamical networks are time-varying generally, in which, the changes of link strengths usually lead to variations of the network topology and coupling configuration $[16,17]$. Therefore, it is meaningful to investigate the synchronization problem of complex dynamical networks with unknown time-varying coupling weights. In order to find the timevarying law of the complex dynamical networks, researchers usually use adaptive methods to estimate the coupling strengths of the network [21]. In paper [5], the author analyzed mean square synchronization of time-delay coupled network, and the unknown periodical coupling weight was estimated successfully with the adaptive leaning update laws and proper controller designed. In 2015, Hao and Li [28] combined adaptive control with learning control to estimate the network unknown periodic coupling structure under the stochastic disturbance successfully. However, the controllers designed in $[5,28]$ are all continuous, the discontinuous method such as intermittent control was not taken into account. Not only that, but also the intrinsic time delay is ubiquitous in neural networks [29], chaotic attractors [30], and even complex dynamical networks [31], which will affect the synchronization performance. So the synchronization of complex dynamical network with time delay is needed to be investigated.

Motivated by the above discussions, we will consider the exponential synchronization problem for time-delay complex dynamical networks with unknown time-varying coupling weights by using an adaptive aperiodically intermittent controller in this paper. The main contribution of our paper is that, combining the advantages of aperiodic intermittent control, adaptive control, and learning control, a new type of aperiodically intermittent synchronization of complex dynamical network is accomplished when the time varying coupling weights among nodes are unknown primitively. The rest of this paper is organized as follows. A new complex dynamical network model is presented, and the problem formulation and preliminaries are presented in Section 2. Section 3 proposes the aperiodically intermittent synchronization approach for complex dynamical networks. In Section 4, a numerical example is given to illustrate the effectiveness of the designed method. Finally, conclusions are presented in Section 5.

\section{Problem Statement and Preliminaries}

In the paper, the time-delay complex dynamical network with unknown periodical outer coupling strengths is given as follows:

$$
\begin{aligned}
\dot{x}_{i}(t)= & f\left(t, x_{i}(t), x_{i}\left(t-\tau_{0}(t)\right)\right) \\
& +\sum_{j=1}^{N} c_{i j}(t) a_{i j} \Gamma x_{j}(t)+u_{i}, \quad i=1,2, \ldots, N .
\end{aligned}
$$

where $x_{i}(t)=\left(x_{i 1}(t), x_{i 2}(t), \ldots, x_{i n}(t)\right)^{T} \in R^{n}$ is the state variable of node $i$ and $f: R^{+} \times R^{n} \times R^{n} \longrightarrow R^{n}, i=1,2, \ldots, N$ is a smooth nonlinear function, describing the local dynamics of each node for network (1). $\tau_{0}(t)$ is the unknown bounded time-varying delay, and there exists a positive constant $\tau_{0}$ such that $0 \leq \tau_{0}(t) \leq \tau_{0}$. The positive definite $\Gamma \in R^{n \times n}$ is the inner coupling matrix. $u_{i}(t)$ is the outer controller to be designed later. The matrix $A=\left(a_{i j}\right)_{N \times N} \in R^{N \times N}$ is the outer coupling matrix, which describes the topology structure of the whole network. And it is defined as follows: if there is a directional connection from node $j$ to node $i(i \neq j)$, then $a_{i j} \neq 0$; otherwise $a_{i j}=0 . c_{i j}(t)$ is the unknown periodical coupling strengths between nodes $i$ and $j$. These elements satisfy the following condition:

$$
c_{i i}(t) a_{i i}=-\sum_{\substack{j=1 \\ j \neq i}}^{N} c_{i j}(t) a_{i j}, \quad i=1,2, \ldots, N .
$$


The initial conditions of network (1) are assumed to be $x_{i}(t)=\psi_{i}(t) \in C\left(\left[-\tau_{0}, 0\right], R^{n}\right)$, where $C\left(\left[-\tau_{0}, 0\right], R^{n}\right)$ represents the set of all $n$-dimensional continuous functions defined on interval $\left[-\tau_{0}, 0\right]$.

Without loss of generality, let the solution $s(t) \in R^{n}$ of (3) be the global exponential synchronization goal orbit:

$$
\dot{s}(t)=f\left(t, s(t), s\left(t-\tau_{0}(t)\right)\right) .
$$

Let $e_{i}(t)=x_{i}(t)-s(t)$; then the error system is

$$
\dot{e}_{i}(t)=\dot{x}_{i}(t)-\dot{s}(t), \quad i=1,2, \ldots, N .
$$

In the paper, we will add proper designed controllers $u_{i}(t), i=1,2, \ldots, N$ intermittently to the dynamical network (1) to realize globally exponential synchronization.

Definition 1. The complex dynamical network (1) is said to be globally exponentially synchronized, if there exist two positive constants $p$ and $q$ such that, for any initial state $x_{i}(t)=\psi_{i}(t) \in C\left(\left[-\tau_{0}, 0\right], R^{n}\right)$, the synchronous error satisfies

$$
\|e(t)\| \leq p e^{-q t}, \quad \forall t \in\left[-\tau_{0},+\infty\right) .
$$

where $e(t)=\left(e_{1}(t), e_{2}(t), \ldots, e_{N}(t)\right)^{T}$.

For more discussion, the following assumptions and lemmas are needed to be introduced firstly.

Assumption 2 (see [21]). For the vector-valued function $f\left(t, x(t), x\left(t-\tau_{0}(t)\right)\right)$, suppose the uniform semi-Lipschitz condition with respect to the time $t$ holds; i.e., for any $x \in$ $R^{n}, y \in R^{n}$, there exists two positive constants $\bar{l}_{1}$ and $\bar{l}_{2}$, such that

$$
\begin{aligned}
& (x(t)-y(t))^{T}\left(f\left(t, x(t), x\left(t-\tau_{0}(t)\right)\right)\right. \\
& \left.\quad-f\left(t, y(t), y\left(t-\tau_{0}(t)\right)\right)\right) \leq \bar{l}_{1}(x(t)-y(t))^{T} \\
& \quad \cdot(x(t)-y(t))+\bar{l}_{2}\left(x\left(t-\tau_{0}(t)\right)-y\left(t-\tau_{0}(t)\right)\right)^{T} \\
& \quad \cdot\left(x\left(t-\tau_{0}(t)\right)-y\left(t-\tau_{0}(t)\right)\right) .
\end{aligned}
$$

Assumption 3. In network (1), the unknown time-varying coupling strengths $c_{i j}(t)$ are periodical parameters; that is, $c_{i j}(t+T)=c_{i j}(t)$ for $t \in[0,+\infty)$, in which $T$ is the known common period of $c_{i j}(t)$.

For further discussion, the aperiodically intermittent control strategy can be expressed as follows. Each controlling cycle $\left[w_{i}, w_{i+1}\right)$ usually contains two types of time zones, one is working time $\left[w_{i}, s_{i}\right)$ and the other is rest time $\left[s_{i}, w_{i+1}\right)$. The controller is activated at each working time and closed at rest time.

As shown in Figure 1, the solid line represents the working time, while the dotted line represents the rest time.

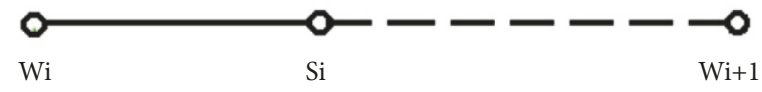

FIGURE 1: Aperiodical intermittent control sketch.

Assumption 4 (see [32]). For the aperiodically intermittent control strategy, there exist two positive scalars $0<\theta<\omega<$ $+\infty$, such that, for $i=0,1,2, \cdots$,

$$
\begin{aligned}
\inf _{i}\left(s_{i}-w_{i}\right) & =\theta, \\
\sup _{i}\left(w_{i+1}-w_{i}\right) & =\omega .
\end{aligned}
$$

Define the maximum proportion of rest width $w_{i+1}-s_{i}$ in the time span $w_{i+1}-w_{i}$ as

$$
\phi=\limsup _{i \rightarrow+\infty} \frac{w_{i+1}-s_{i}}{w_{i+1}-w_{i}} .
$$

Lemma 5 (see [32]). For any $i=0,1,2, \ldots$, if we denote

$$
\phi(t)=\frac{t-s_{i}}{t-w_{i}}, \quad t \in\left[s_{i}, w_{i+1}\right] .
$$

Then $\phi(t)$ is an strictly increasing function, so $\phi(t) \leq \phi\left(w_{i+1}\right)=$ $\left(w_{i+1}-s_{i}\right) /\left(w_{i+1}-w_{i}\right)$.

Lemma 6 (see $[32,33])$. Suppose that function $y(t)$ is continuous and nonnegative for $t \longrightarrow[-\tau,+\infty)$ and satisfies the following condition:

$$
\begin{array}{ll}
\dot{y}(t) \leq-\gamma_{1} y(t)+\gamma_{2}\left(\sup _{t-\tau \leq s \leq t} y(s)\right), & w_{i} \leq t \leq s_{i}, \\
\dot{y}(t) \leq \gamma_{3} y(t)+\gamma_{4}\left(\sup _{t-\tau \leq s \leq t} y(s)\right), & s_{i} \leq t \leq w_{i+1} .
\end{array}
$$

where $\gamma_{1}, \gamma_{2}, \gamma_{3}, \gamma_{4}$ are positive constants and $i=0,1,2, \cdots$. Suppose that, for the aperiodically intermittent control, there exists a constant $\phi$ defined in (8). If

$$
\begin{aligned}
& \gamma_{1}>\gamma^{*}=\max \left\{\gamma_{2}, \gamma_{4}\right\}>0, \\
& \rho=\gamma_{1}+\gamma_{3}>0, \\
& \omega=\lambda-\rho \phi>0,
\end{aligned}
$$

then

$$
y(t) \leq\left(\sup _{-\tau \leq s \leq 0} y(s)\right) \exp \{-\omega t\}, \quad t \geq 0,
$$

where $\lambda>0$ is the unique positive solution of the equation $\lambda-$ $\gamma_{1}+\gamma^{*} \exp \{\lambda \tau\}=0$.

\section{Aperiodically Intermittent Synchronization for Complex Dynamical Networks}

In this section, we consider the synchronization problem of coupled complex dynamical network (1) via aperiodically 
intermittent control. In order to achieve the synchronization objective (3), we choose the adaptive controller $u_{i}(t)$ for the $i$-th node as follows:

$$
\begin{aligned}
& u_{i}(t) \\
& =\left\{\begin{array}{lr}
-k_{i}(t) \Gamma e_{i}(t)-\sum_{j=1}^{N} \widehat{c}_{i j}(t) a_{i j} \Gamma e_{j}(t), & t \in\left[w_{i}, s_{i}\right), \\
0, & t \in\left[s_{i}, w_{i+1}\right), \\
1 \leq i \leq N .
\end{array}\right.
\end{aligned}
$$

The update law of the adaptive parameter $k_{i}(t), i=$ $1,2, \ldots, N$ is

$$
\dot{k}_{i}(t)= \begin{cases}\alpha_{i} \exp \left(\beta_{1} t\right) e_{i}^{T}(t) \Gamma e_{i}(t), & t \in\left[w_{i}, s_{i}\right), \\ 0, & t \in\left[s_{i}, w_{i+1}\right) .\end{cases}
$$

where $\alpha_{i}$ and $\beta_{1}$ are positive constants.

For the unknown time-varying periodical coupling strengths $c_{i j}(t), i, j=1,2, \ldots, N$, the parameters estimation are designed by

$$
\widehat{c}_{i j}(t)= \begin{cases}\widehat{c}_{i j}(t-T)+e_{i j}^{*} a_{i j} e_{i}^{T}(t) \Gamma e_{j}(t), & t \in[k T,(k+1) T), k=1,2, \cdots \\ e_{i j}(t) a_{i j} e_{i}^{T}(t) \Gamma e_{j}(t), & t \in[0, T) \\ 0, & t \in[-T, 0) .\end{cases}
$$

where $\widehat{c}_{i j}(t)$ is the estimation of coupling strength $c_{i j}(t)$. Denote $\widetilde{c}_{i j}(t)=c_{i j}(t)-\widehat{c}_{i j}(t)$ is the estimation error. $\varrho_{i j}^{*}$ are positive constants, $\varrho_{i j}(t)$ is a continuous and strictly increasing function for $t \in[0, T]$ and satisfies $\varrho_{i j}(0)=$ $0, \varrho_{i j}(T)=\varrho_{i j}^{*}$.

Basing on the designed controllers, the following synchronization error system can be obtained:

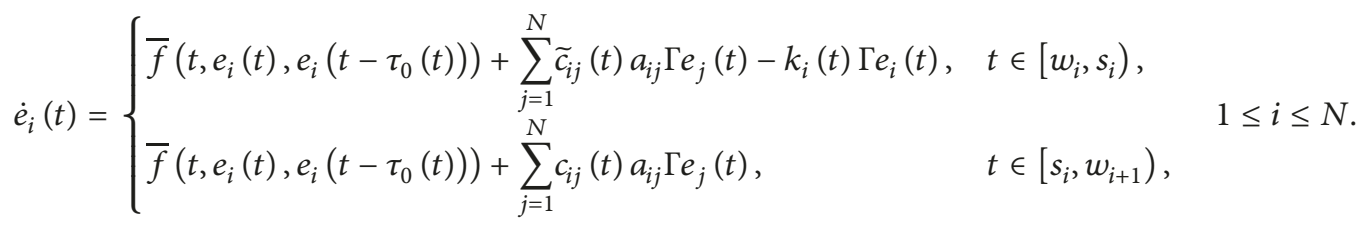

where $\bar{f}\left(t, e_{i}(t), e_{i}\left(t-\tau_{0}(t)\right)\right)=f\left(t, x_{i}(t), x_{i}\left(t-\tau_{0}(t)\right)\right)-$ $f\left(t, s(t), s\left(t-\tau_{0}(t)\right)\right)$.

Obviously, we can see that exponential synchronization of complex dynamical network (1) with controller $u_{i}$ equals the exponential stability of error system (16). So, next, a sufficient condition for the controlled complex network realizing global exponential synchronization will be presented as follows.

Theorem 7. Suppose that Assumptions 2-4 hold. If there exist positive constants $\beta_{1}$ and $\beta_{2}\left(\beta_{2}>\beta_{1}\right)$, such that the following conditions hold,

$$
\begin{aligned}
\eta_{2}+\lambda_{\max }\left(\widehat{A}_{C}^{s}\right) & <0, \\
\beta_{1}-2 l_{2} & >0, \\
\xi-\beta_{2} \phi & >0 .
\end{aligned}
$$

where $\xi>0$ is the unique positive solution of equation $\xi-$ $\beta_{1}+l \exp \left\{\xi \tau_{0}\right\}=0, l=\max \left\{l_{2}, l_{3}\right\}$, then the controlled network (1) with adaptive periodical outer coupling update laws (15) is globally exponentially synchronized under the adaptive aperiodically intermittent controllers (13) and (14).
Proof. Construct the Lyapunov-Krasovskii-like function candidate as follows:

$$
\begin{aligned}
V(t)= & \frac{1}{2} \sum_{i=1}^{N} e_{i}^{T}(t) e_{i}(t)+\frac{1}{2} \sum_{i=1}^{N} \sum_{j=1}^{N} \frac{1}{e_{i j}^{*}} \int_{t-T}^{t} \tilde{c}_{i j}^{2}(\tau) d \tau \\
& +\frac{1}{2} \sum_{i=1}^{N} \exp \left(-\beta_{1} t\right) \frac{\left(k_{i}(t)-k_{i}^{*}\right)^{2}}{\alpha_{i}},
\end{aligned}
$$

where $t \geq T, k_{i}^{*}$ are some sufficiently large positive constants which will be determined later.

When $t \in\left[w_{i}, s_{i}\right)$, taking the derivative along the trajectories of the error system, we get

$$
\begin{aligned}
\dot{V}(t) & =\sum_{i=1}^{N} e_{i}^{T}(t) \dot{e}_{i}(t)+\frac{1}{2} \sum_{i=1}^{N} \sum_{j=1}^{N} \frac{1}{e_{i j}^{*}}\left(\tilde{c}_{i j}^{2}(t)-\tilde{c}_{i j}^{2}(t-T)\right) \\
- & \frac{\beta_{1}}{2} \sum_{i=1}^{N} \exp \left(-\beta_{1} t\right) \frac{\left(k_{i}(t)-k_{i}^{*}\right)^{2}}{\alpha_{i}} \\
+ & \sum_{i=1}^{N} \exp \left(-\beta_{1} t\right) \frac{\left(k_{i}(t)-k_{i}^{*}\right)}{\alpha_{i}} \cdot \dot{k}_{i}(t)=\sum_{i=1}^{N} e_{i}^{T}(t)
\end{aligned}
$$




$$
\begin{aligned}
& \cdot\left(\bar{f}\left(t, e_{i}(t), e_{i}\left(t-\tau_{0}(t)\right)\right)+\sum_{j=1}^{N} \widetilde{c}_{i j}(t) a_{i j} \Gamma e_{j}(t)\right) \\
& -\frac{\beta_{1}}{2} \sum_{i=1}^{N} \exp \left(-\beta_{1} t\right) \frac{\left(k_{i}(t)-k_{i}^{*}\right)^{2}}{\alpha_{i}} \\
& +\sum_{i=1}^{N} \exp \left(-\beta_{1} t\right) \frac{\left(k_{i}(t)-k_{i}^{*}\right)}{\alpha_{i}} \cdot \dot{k}_{i}(t)+\frac{1}{2} \\
& \cdot \sum_{i=1}^{N} \sum_{j=1}^{N} \frac{1}{Q_{i j}^{*}}\left(\widetilde{c}_{i j}^{2}(t)-\widetilde{c}_{i j}^{2}(t-T)\right)-\sum_{i=1}^{N} e_{i}^{T}(t) k_{i}(t) \\
& \cdot \Gamma e_{i}(t) .
\end{aligned}
$$

Next we will calculate the items separately. Combining with Assumption 2, we can have

$$
\begin{aligned}
& e_{i}^{T}(t) \bar{f}\left(t, e_{i}(t), e_{i}\left(t-\tau_{0}(t)\right)\right) \\
& \quad \leq \bar{l}_{1} e^{T}(t) e(t)+\bar{l}_{2} e^{T}\left(t-\tau_{0}(t)\right) e\left(t-\tau_{0}(t)\right) .
\end{aligned}
$$

Considering the adaptive law (14), one can have

$$
\begin{gathered}
\sum_{i=1}^{N} \exp \left(-\beta_{1} t\right) \frac{\left(k_{i}(t)-k_{i}^{*}\right)}{\alpha_{i}} \cdot \dot{k}_{i} \\
=\sum_{i=1}^{N}\left(k_{i}(t)-k_{i}^{*}\right) e_{i}^{T}(t) \Gamma e_{i}(t) .
\end{gathered}
$$

Using the matrix equality $(a-b)^{T} H(a-b)-(a-c)^{T} H(a-$ $c)=(c-b)^{T} H[2(a-b)+(b-c)]$ and the estimation of unknown coupling item (15), we can obtain

$$
\begin{gathered}
\sum_{i=1}^{N} \sum_{j=1}^{N} \frac{1}{\varrho_{i j}^{*}}\left(\widetilde{c}_{i j}^{2}(t)-\widetilde{c}_{i j}^{2}(t-T)\right) \\
=-\sum_{i=1}^{N} \sum_{j=1}^{N} \frac{1}{\varrho_{i j}^{*}}\left(\widehat{c}_{i j}(t)-\widehat{c}_{i j}(t-T)\right)^{2} \\
-2 \sum_{i=1}^{N} \sum_{j=1}^{N} \frac{1}{\varrho_{i j}^{*}}\left(c_{i j}(t)-\widehat{c}_{i j}(t-T)\right) \\
\cdot\left(\widehat{c}_{i j}(t)-\widehat{c}_{i j}(t-T)\right) \\
=-2 \sum_{i=1}^{N} \sum_{j=1}^{N} \widetilde{c}_{i j}(t) a_{i j} e_{i}^{T}(t) \Gamma e_{j}(t) \\
-\sum_{i=1}^{N} \sum_{j=1}^{N} \frac{1}{e_{i j}^{*}}\left(\widehat{c}_{i j}(t)-\widehat{c}_{i j}(t-T)\right)^{2} \\
\leq-2 \sum_{i=1}^{N} \sum_{j=1}^{N} \widetilde{c}_{i j}(t) a_{i j} e_{i}^{T}(t) \Gamma e_{j}(t) .
\end{gathered}
$$

With the above results and properties of Kronecker production, substitute (22), (23), and (24) into (21), one gets

$$
\begin{aligned}
& \dot{V}(t) \leq \bar{l}_{1} e^{T}(t) e(t)+\bar{l}_{2} e^{T}\left(t-\tau_{0}(t)\right) e\left(t-\tau_{0}(t)\right) \\
&-\sum_{i=1}^{N} e_{i}^{T}(t) k_{i}^{*} \Gamma e_{i}(t)-\frac{\beta_{1}}{2} \\
& \cdot \sum_{i=1}^{N} \exp \left(-\beta_{1} t\right) \frac{\left(k_{i}(t)-k_{i}^{*}\right)^{2}}{\alpha_{i}} \leq \eta_{1} e^{T}(t)\left(I_{N} \otimes \Gamma\right) \\
& \cdot e(t)+\bar{l}_{2} e^{T}\left(t-\tau_{0}(t)\right) e\left(t-\tau_{0}(t)\right)-e^{T}(t)\left(K^{*}\right. \\
&\otimes \Gamma) e(t)-\frac{\beta_{1}}{2}\left(\sum_{i=1}^{N} e_{i}^{T}(t) e_{i}(t)\right. \\
&\left.+\sum_{i=1}^{N} \exp \left(-\beta_{1} t\right) \frac{\left(k_{i}(t)-k_{i}^{*}\right)^{2}}{\alpha_{i}}\right) \leq e^{T}(t) \\
& \cdot\left(\left(R-K^{*}\right) \otimes \Gamma\right) e(t)+\bar{l}_{2} e^{T}\left(t-\tau_{0}(t)\right) e(t \\
&\left.-\tau_{0}(t)\right)+\frac{\beta_{1}}{2} \sum_{i=1}^{N} \sum_{j=1}^{N} \frac{1}{e_{i j}^{*}} \int_{t-T}^{t} \tau_{i j}^{2}(\tau) d \tau \\
&+\frac{\beta_{1}}{2}\left(\sum_{i=1}^{N} e_{i}^{T}(t) e_{i}(t)\right. \\
&+\sum_{i=1}^{N} \exp \left(-\beta_{1} t\right) \frac{\left(k_{i}(t)-k_{i}^{*}\right)^{2}}{\alpha_{i}} \\
&\left.e_{i j}^{*} \int_{t-T} \tilde{c}_{i j}^{2}(\tau) d \tau\right), \\
&
\end{aligned}
$$

where the vectors $e(t)=\left(e_{1}(t), e_{2}(t), \ldots, e_{N}(t)\right)^{T}, e\left(t-\tau_{0}(t)\right)=$ $\left(e_{1}\left(t-\tau_{0}(t)\right), e_{2}\left(t-\tau_{0}(t)\right), \ldots, e_{N}\left(t-\tau_{0}(t)\right)\right)^{T}, \eta_{1}=\bar{l}_{1} / \lambda_{\text {min }}(\Gamma)+$ $\beta_{1} / 2, R=\eta_{1} I_{N}, K^{*}=\operatorname{diag}\left\{k_{1}^{*}, \cdots, k_{N}^{*}\right\}$.

So we can see that if we choose $k_{i}^{*}>\eta_{1}$, the matrix $R-$ $K^{*}<0$. Let $l_{2}=\max \left\{2 \bar{l}_{2}, \beta_{1}\right\}$; then we can acquire

$$
\dot{V}(t) \leq-\beta_{1} V(t)+l_{2}\left(\sup _{t-\tau_{0} \leq \varsigma \leq t} V(\varsigma)\right)
$$

When $t \in\left[s_{i}, w_{i+1}\right)$, taking the derivative along the trajectories of the error system (16), we have

$$
\begin{gathered}
\dot{V}(t) \leq \bar{l}_{1} e^{T}(t) e(t)+\bar{l}_{2} e^{T}\left(t-\tau_{0}(t)\right) e\left(t-\tau_{0}(t)\right) \\
+\sum_{i=1}^{N} \sum_{j=1}^{N} \widehat{c}_{i j}(t) e_{i}^{T}(t) a_{i j} \Gamma e_{j}(t)-\frac{\beta_{1}}{2}
\end{gathered}
$$




$$
\begin{aligned}
& \cdot \sum_{i=1}^{N} \exp \left(-\beta_{1} t\right) \frac{\left(k_{i}(t)-k_{i}^{*}\right)^{2}}{\alpha_{i}} \leq \eta_{2} e^{T}(t)\left(I_{N} \otimes \Gamma\right) \\
& \cdot e(t)+\bar{l}_{2} e^{T}\left(t-\tau_{0}(t)\right) e\left(t-\tau_{0}(t)\right)+e^{T}(t)\left(\widehat{A}_{C}^{s}\right. \\
& \otimes \Gamma) e(t)+\frac{\beta_{2}-\beta_{1}}{2}\left(\sum_{i=1}^{N} e_{i}^{T}(t) e_{i}(t)\right. \\
& \left.+\sum_{i=1}^{N} \exp \left(-\beta_{1} t\right) \frac{\left(k_{i}(t)-k_{i}^{*}\right)^{2}}{\alpha_{i}}\right) \leq e^{T}(t)(\widetilde{R} \otimes \Gamma) \\
& \cdot e(t)+\bar{l}_{2} e^{T}\left(t-\tau_{0}(t)\right) e\left(t-\tau_{0}(t)\right) \\
& +\frac{\beta_{2}-\beta_{1}}{2}\left(\sum_{i=1}^{N} e_{i}^{T}(t) e_{i}(t)\right. \\
& +\sum_{i=1}^{l} \exp \left(-\beta_{1} t\right) \frac{\left(k_{i}(t)-k_{i}^{*}\right)^{2}}{\alpha_{i}} \\
& \left.+\sum_{i=1}^{N} \sum_{j=1}^{N} \frac{1}{\varrho_{i j}^{*}} \int_{t-T}^{t} \widetilde{c}_{i j}^{2}(\tau) d \tau\right),
\end{aligned}
$$

where $\widetilde{R}=\eta_{2} I_{N}+\widehat{A}_{C}^{s}, \widehat{A}_{C}^{s}=\left(\widehat{A}_{C}+\widehat{A}_{C}^{T}\right) / 2, \widehat{A}_{C}=\left(c_{i j}(t)\right.$. $\left.a_{i j}\right)_{N \times N}, \eta_{2}=\bar{l}_{1} / \lambda_{\text {min }}(\Gamma)-\left(\beta_{2}-\beta_{1}\right) / 2$. According to Lemma 6 , it follows that $\lambda_{\max }(\widetilde{R}) \leq \eta_{2}+\lambda_{\max }\left(\widehat{A}_{C}^{s}\right)$. If condition (17) is satisfied, it is easy to have $\widetilde{R}<0$. Let $l_{3}=2 \bar{l}_{2}$. Then we can have

$$
\dot{V}(t) \leq\left(\beta_{2}-\beta_{1}\right) V(t)+l_{3}\left(\sup _{t-\tau_{0} \leq \varsigma \leq t} V(\varsigma)\right) .
$$

Thus, we obtain

$$
\begin{aligned}
& \dot{V}(t) \leq-\beta_{1} V(t)+l_{2}\left(\sup _{t-\tau_{0} \leq \varsigma \leq t} V(\varsigma)\right), \quad t \in\left[w_{i}, s_{i}\right), \\
& \dot{V}(t) \leq\left(\beta_{2}-\beta_{1}\right) V(t)+l_{3}\left(\sup _{t-\tau_{0} \leq \varsigma \leq t} V(\varsigma)\right), \\
& t \in\left[s_{i}, w_{i+1}\right) .
\end{aligned}
$$

Combining Lemma 6 with conditions (18) and (19), we can get $V(t) \leq\left(\sup _{t-\tau_{0} \leq \varsigma \leq t} V(\varsigma)\right) \exp (-\eta t)$ for $t \geq 0$ immediately, where $\eta=\xi-\left(l+\beta_{2}-\beta_{1}\right) \phi$ and the parameter $\xi$ satisfies the equation $\xi-\beta_{1}+l \exp \left\{\xi \tau_{0}\right\}=0$. So the zero solution of the error system (15) is globally exponentially stable. According to the calculation of $V(t)$, we can obtain $\|e(t)\|^{2} \leq 2\|V(t)\|$; i.e., $\|e(t)\| \leq \sqrt{2}\|V(t)\|^{1 / 2}$. And hence $\|e(t)\| \leq \sqrt{2\left(\sup _{t-\tau_{0} \leq \varsigma \leq t} V(\varsigma)\right)} \exp (-(\eta / 2) t)$ holds, which implies the global exponential synchronization is achieved. Thus the proof is completed.
If the complex dynamical network (1) has no any internal time delay, i.e., $\tau_{0}(t)=0$, the system (1) can be rewritten as

$$
\dot{x}_{i}(t)=f\left(t, x_{i}(t)\right)+\sum_{j=1}^{N} c_{i j}(t) a_{i j} \Gamma x_{j}(t)+u_{i},
$$

$$
i=1,2, \ldots, N \text {. }
$$

and the synchronization target orbit is described by $\dot{s}(t)=$ $f(t, s(t))$. In this case, we can see that $\bar{l}_{2}=0$ holds. By using a similar line of arguments as that in Theorem 7 , the following result is easily achieved.

Corollary 8. Suppose that Assumptions 2-4 hold. If there exist positive constants $\beta_{1}$ and $\beta_{2}\left(\beta_{2}>\beta_{1}\right)$, such that the following conditions hold,

$$
\begin{array}{r}
\eta_{2}+\lambda_{\max }\left(\widehat{A}_{C}^{s}\right)<0, \\
\xi-\beta_{2} \phi>0,
\end{array}
$$

then the controlled network (1) with adaptive periodical outer coupling update laws (15) is globally exponential synchronized under the adaptive aperiodically intermittent controllers (13) and (14).

Remark 9. When $\bar{l}_{2}=0$, the equation is $\xi-\beta_{1}+l_{3} \exp \left\{\xi \tau_{0}\right\}=$ 0 , so inequality (19) can be expressed as (32).

\section{Numerical Simulations}

In this section, we will illustrate the effectiveness of the proposed approach to achieve globally exponential synchronization of complex networks (1) with unknown time-varying coupling strengths via aperiodically intermittent control. Without loss of generality, we choose the delayed Chua's chaotic models as the nodes' dynamics. The chaotic Chua's system dynamical function is as follows:

$$
\begin{gathered}
\left(\begin{array}{c}
\dot{x}_{1}(t) \\
\dot{x}_{2}(t) \\
\dot{x}_{3}(t)
\end{array}\right)=\left(\begin{array}{ccc}
-\alpha\left(1+m_{2}\right) & \alpha & 0 \\
1 & -1 & 1 \\
0 & -\beta & -\omega
\end{array}\right)\left(\begin{array}{l}
x_{1}(t) \\
x_{2}(t) \\
x_{3}(t)
\end{array}\right) \\
+\left(\begin{array}{c}
0 \\
0 \\
-\beta \zeta \sin \left(v x_{1}\left(t-\tau_{1}(t)\right)\right)
\end{array}\right) \\
+\left(\begin{array}{c}
-\frac{1}{2} \alpha\left(m_{1}-m_{2}\right)\left(\left|x_{1}(t)+1\right|-\left|x_{1}(t)-1\right|\right) \\
0 \\
0
\end{array}\right) .
\end{gathered}
$$

where the parameters are $\alpha=10, \beta=18.53, \omega=$ $0.1636, m_{1}=-1.4325, m_{2}=-0.7831, \zeta=0.2, v=0.5$, respectively, and the time delay $\tau_{1}(t)=0.02$. By calculation [9], we have $\bar{l}_{1}=11.6435, \bar{l}_{2}=0.3088$. 
The outer coupling matrix which depicts the structure of network (1) is a $10 \times 10$ matrix, which is chosen as follows:

A

$$
=\left(\begin{array}{cccccccccc}
-3 & 0 & 1.5 & 0 & 0.5 & 0 & 0.5 & 0 & 0 & 0.5 \\
0 & -2 & 0 & 0.3 & 0.5 & 0 & 0 & 0.7 & 0 & 0.5 \\
0 & 1 & -4 & 0 & 0 & 1 & 0 & 1 & 0 & 1 \\
1 & 0 & 0 & -3 & 0 & 0 & 0.5 & 0.5 & 0 & 1 \\
0.2 & 0 & 0.5 & 0 & -3 & 0.5 & 0 & 0.8 & 1 & 0 \\
0 & 1 & 0 & 0 & 1 & -4 & 0 & 0 & 0 & 2 \\
0 & 1 & 1 & 0 & 2 & 0 & -5 & 0 & 0 & 1 \\
1 & 0 & 0.5 & 1.5 & 0 & 0 & 0.5 & -4.5 & 1 & 0 \\
0.5 & 0 & 1 & 0 & 1 & 1 & 0 & 0.5 & -4 & 0 \\
0 & 1 & 0.5 & 0 & 0 & 0 & 1 & 0 & 0 & -2.5
\end{array}\right) .
$$

The coupling weight is

$$
\begin{aligned}
& c_{11}(t)=\frac{1}{6}+\frac{1}{6} \sin \pi t-\frac{13}{60} \cos \frac{\pi}{2} t, \\
& c_{12}(t)=2 \text {, } \\
& c_{13}(t)=0 \text {, } \\
& c_{14}(t)=2.8 \text {, } \\
& c_{15}(t)=1-1.3 \cos \frac{\pi}{2} t, \\
& c_{16}(t)=-0.5 \cos \frac{\pi}{2} t, \\
& c_{17}(t)=0 \text {, } \\
& c_{18}(t)=\sin \pi t, \\
& c_{19}(t)=0 \text {, } \\
& c_{110}(t)=\sin \pi t ; \\
& c_{21}(t)=1 \text {, } \\
& c_{22}(t)=\frac{1}{2}, \\
& c_{23}(t)=\cos \pi t, \\
& c_{24}(t)=0 \text {, } \\
& c_{25}(t)=0 \text {, } \\
& c_{26}(t)=0.5 \cos \frac{\pi}{3} t, \\
& c_{27}(t)=\cos \pi t, \\
& c_{28}(t)=0 \text {, } \\
& c_{29}(t)=\cos \frac{\pi}{3} t,
\end{aligned}
$$

$$
\begin{aligned}
& c_{210}(t)=1 \\
& c_{31}(t)=1-\sin \frac{\pi}{3} t, \\
& c_{32}(t)=1+0.5 \cos \frac{\pi}{3} t, \\
& c_{33}(t)=\frac{1}{2}+\frac{1}{2} \cos \frac{\pi}{3} t+\frac{1}{4} \sin \frac{\pi}{3} t, \\
& c_{34}(t)=0 \text {, } \\
& c_{35}(t)=0.7 \text {, } \\
& c_{36}(t)=1+1.5 \cos \frac{\pi}{3} t, \\
& c_{37}(t)=\cos \frac{\pi}{3} t, \\
& c_{38}(t)=\sin \frac{\pi}{3} t, \\
& c_{39}(t)=-1 \text {, } \\
& c_{310}(t)=0 \text {; } \\
& c_{41}(t)=1+\cos \frac{\pi}{3} t, \\
& c_{42}(t)=\sin \pi t, \\
& c_{43}(t)=2 \sin \pi t, \\
& c_{44}(t)=\frac{1}{6}+\frac{2}{3} \cos \frac{\pi}{3} t+\frac{1}{3} \sin \pi t, \\
& c_{45}(t)=0 \text {, } \\
& c_{46}(t)=\cos \frac{\pi}{3} t, \\
& c_{47}(t)=\sin \pi t, \\
& c_{48}(t)=-1+\sin \pi t, \\
& c_{49}(t)=-\cos \frac{\pi}{3} t, \\
& c_{410}(t)=\cos \frac{\pi}{3} t \\
& c_{51}(t)=0 \text {, } \\
& c_{52}(t)=0 \text {, } \\
& c_{53}(t)=1 \text {, } \\
& c_{54}(t)=0 \text {, } \\
& c_{55}(t)=\frac{1}{3}-\frac{11}{12} \cos \frac{2 \pi}{3} t, \\
& c_{56}(t)=1+0.5 \cos \frac{2 \pi}{3} t \text {, } \\
& c_{57}(t)=0 \text {, } \\
& c_{58}(t)=0 \text {, }
\end{aligned}
$$




$$
\begin{aligned}
& c_{59}(t)=-3 \cos \frac{2 \pi}{3} t \\
& c_{510}(t)=2.3+\cos \frac{2 \pi}{3} t \\
& c_{61}(t)=-2.5-1.4 \sin \frac{\pi}{3} t-1.3 \cos \frac{2 \pi}{3} t, \\
& c_{62}(t)=0.1+0.1 \sin \frac{\pi}{3} t, \\
& c_{63}(t)=0 \text {, } \\
& c_{64}(t)=0 \text {, } \\
& c_{65}(t)=1-\cos \frac{2 \pi}{3} t \\
& c_{66}(t)=\frac{11}{40}+\frac{9}{40} \sin \frac{\pi}{3} t-\frac{1}{4} \cos \frac{2 \pi}{3} t, \\
& c_{67}(t)=0 \text {, } \\
& c_{68}(t)=1+0.5 \sin \frac{\pi}{3} t, \\
& c_{69}(t)=0, \\
& c_{610}(t)=0.5 \sin \frac{\pi}{3} t \\
& c_{71}(t)=1, \\
& c_{72}(t)=-5-\cos \pi t, \\
& c_{73}(t)=1+\cos \pi t, \\
& c_{74}(t)=0 \text {, } \\
& c_{75}(t)=0 \text {, } \\
& c_{76}(t)=0.5 \cos \frac{\pi}{3} t, \\
& c_{77}(t)=-\frac{4}{5}-\frac{1}{10} \cos \frac{\pi}{3} t, \\
& c_{78}(t)=2 \cos \pi t, \\
& c_{79}(t)=0 \text {, } \\
& c_{710}(t)=-0.5 \cos \frac{\pi}{3} t ; \\
& c_{81}(t)=1-\sin \pi t, \\
& c_{82}(t)=1+1.5 \cos \pi t, \\
& c_{83}(t)=0 \text {, } \\
& c_{84}(t)=0 \text {, } \\
& c_{85}(t)=0.7, \\
& c_{86}(t)=1-0.5 \cos \pi t, \\
& c_{87}(t)=0 \text {, } \\
& c_{88}(t)=\frac{2}{9}-\frac{2}{9} \sin \pi t, \\
& c_{89}(t)=0 \text {, }
\end{aligned}
$$

$$
\begin{aligned}
& c_{810}(t)=-\cos \pi t \\
& c_{91}(t)=1+\cos \frac{\pi}{3} t, \\
& c_{92}(t)=\sin \pi t, \\
& c_{93}(t)=-2 \sin \pi t \text {, } \\
& c_{94}(t)=-1 \text {, } \\
& c_{95}(t)=0 \text {, } \\
& c_{96}(t)=3-0.5 \cos \frac{\pi}{3} t, \\
& c_{97}(t)=\sin \pi t, \\
& c_{98}(t)=0 \text {, } \\
& c_{99}(t)=\frac{7}{8}-\frac{1}{2} \sin \pi t, \\
& c_{910}(t)=-\cos \frac{\pi}{3} t ; \\
& c_{101}(t)=0 \text {, } \\
& c_{102}(t)=\cos \frac{\pi}{2} t \text {, } \\
& c_{103}(t)=1-0.5 \cos \frac{\pi}{2} t, \\
& c_{104}(t)=-\cos \frac{\pi}{2} t, \\
& c_{105}(t)=-1-2 \cos \frac{2 \pi}{3} t, \\
& c_{106}(t)=1+0.5 \cos \frac{\pi}{2} t, \\
& c_{107}(t)=-\sin \pi t, \\
& c_{108}(t)=\sin \pi t, \\
& c_{109}(t)=-\sin \pi t, \\
& c_{1010}(t)=-\frac{1}{5}-\frac{2}{5} \sin \pi t+\frac{3}{10} \cos \frac{\pi}{2} t \text {. }
\end{aligned}
$$

By calculation, we get the common period $T=6$ of $c_{i j}(t)$ and the parameters $\Omega^{*}=\left(\varrho_{i j}^{*}\right)_{10 \times 10}$ are designed as

$$
\begin{aligned}
& \Omega^{*}=\left(e_{i j}^{*}\right) \\
& =\left(\begin{array}{cccccccccc}
1.5 & 0.3 & 0.7 & 2 & 1.1 & 0.1 & 1.7 & 0.2 & 0.1 & 0.4 \\
0.1 & 0.2 & 0.4 & 0.5 & 0.6 & 0.3 & 0.6 & 0.2 & 0.9 & 2.1 \\
0.9 & 0.8 & 0.5 & 0.7 & 1.1 & 0.5 & 0.5 & 0.4 & 0.2 & 1.1 \\
0.5 & 1.5 & 0.9 & 2.1 & 1.8 & 0.6 & 0.8 & 0.6 & 0.8 & 1.6 \\
1.2 & 1.4 & 1.8 & 2 & 2.2 & 0.2 & 0.2 & 1.0 & 0.8 & 0.1 \\
0.5 & 1.3 & 1.7 & 1.2 & 0.1 & 0.4 & 0.1 & 0.3 & 0.3 & 0.9 \\
0.1 & 0.8 & 0.4 & 0.5 & 0.9 & 1.1 & 0.7 & 0.6 & 0.5 & 0.4 \\
0.9 & 0.2 & 1.5 & 0.7 & 1.4 & 0.6 & 0.6 & 0.9 & 0.5 & 0.7 \\
0.8 & 1.1 & 0.1 & 0.1 & 0.8 & 0.3 & 0.5 & 0.7 & 1.1 & 1.3 \\
0.2 & 0.4 & 0.8 & 1.0 & 1.2 & 0.8 & 0.1 & 1.2 & 0.1 & 1.7
\end{array}\right),
\end{aligned}
$$




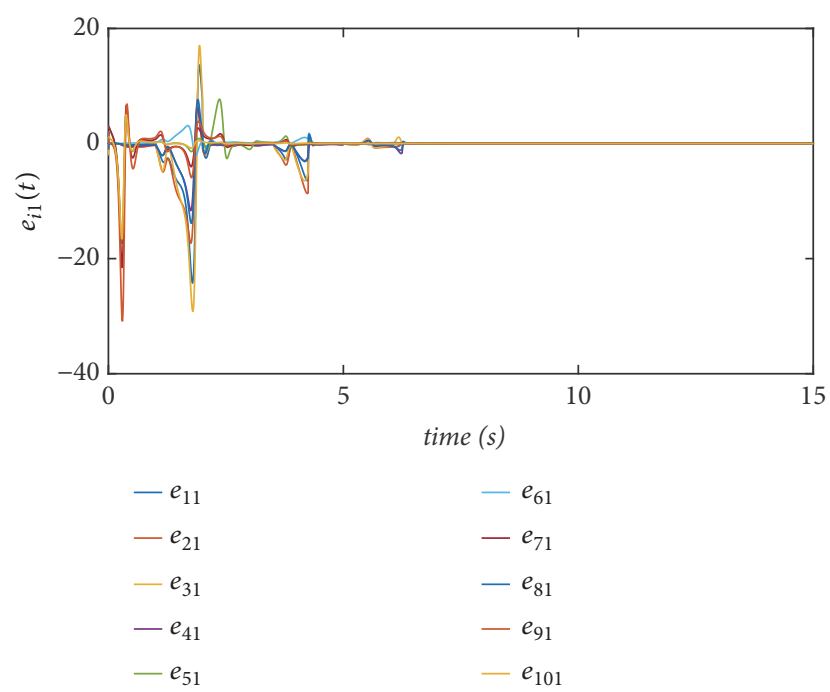

FIgURE 2: The first component of error evolution for each node.

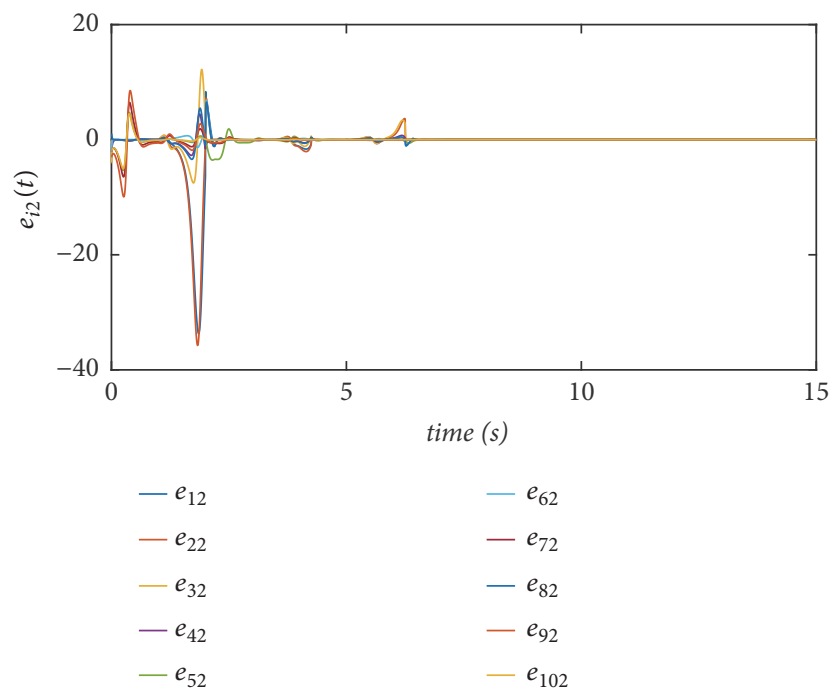

Figure 3: The second component of error evolution for each node.

and $\varrho_{i j}(t)=(t / 6) \varrho_{i j}^{*}$ for $i, j=1,2, \ldots, 10$. For simplicity, the inner coupling matrices are designed as follows: $\Gamma=$ $\operatorname{diag}\{0.5,1,0.8\}$. And the initial values of target orbit are $s(0)=(-0.2,0.2,0.5)$.

The dynamical network with ten nodes can be expressed as follows:

$$
\begin{aligned}
\dot{x}_{i}(t)= & f\left(t, x_{i}(t), x_{i}\left(t-\tau_{0}(t)\right)\right)+\sum_{j=1}^{10} c_{i j}(t) a_{i j} \Gamma x_{j}(t) \\
& +u_{i}, \quad i=1,2, \ldots, 10 .
\end{aligned}
$$

Choosing initial values of complex dynamical network (37) randomly in $[-5,5]$, for the time span $[0,15]$, the work time is $[0,2) \cup[4,7) \cup[10,12.5)$, and the rest time is $[2,4) \cup$ $[7,10) \cup[12.5,15)$. With aperiodically intermittent controller (13), (14), and adaptive update law (15), the synchronization simulations of the complex dynamical network (37) are shown in Figures 2-9.

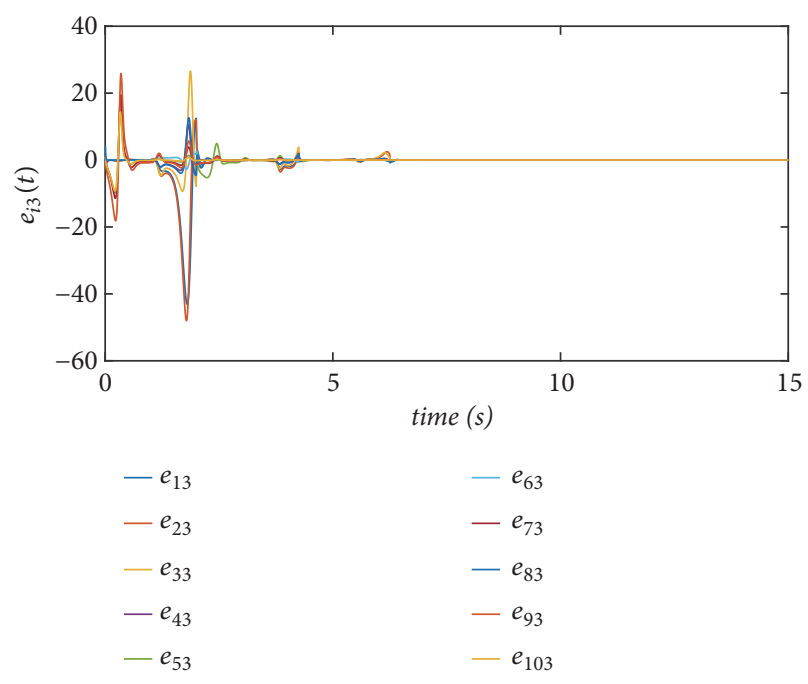

FIGURE 4: The third component of error evolution for each node.

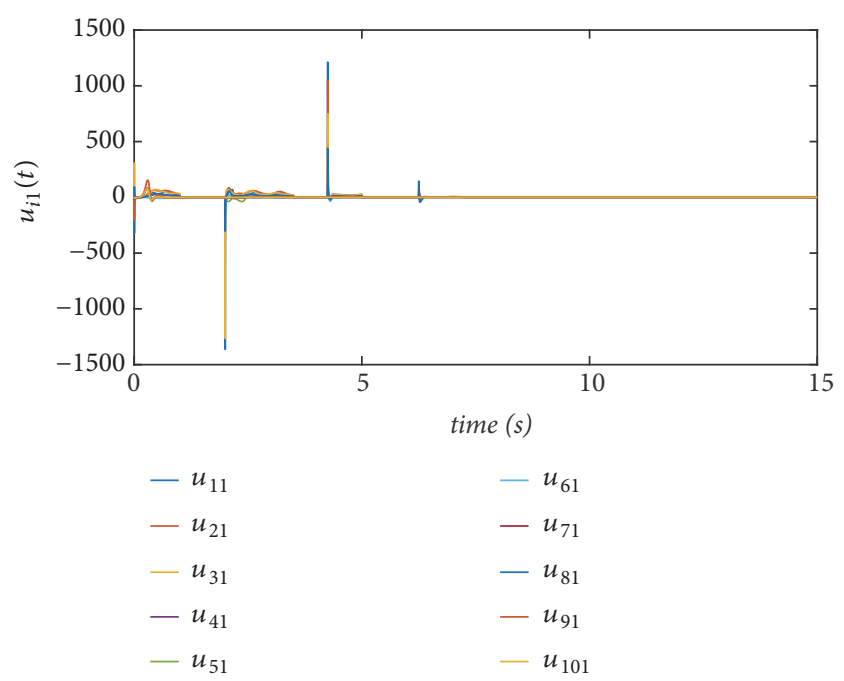

FIGURE 5: The first component of the controller for each node.
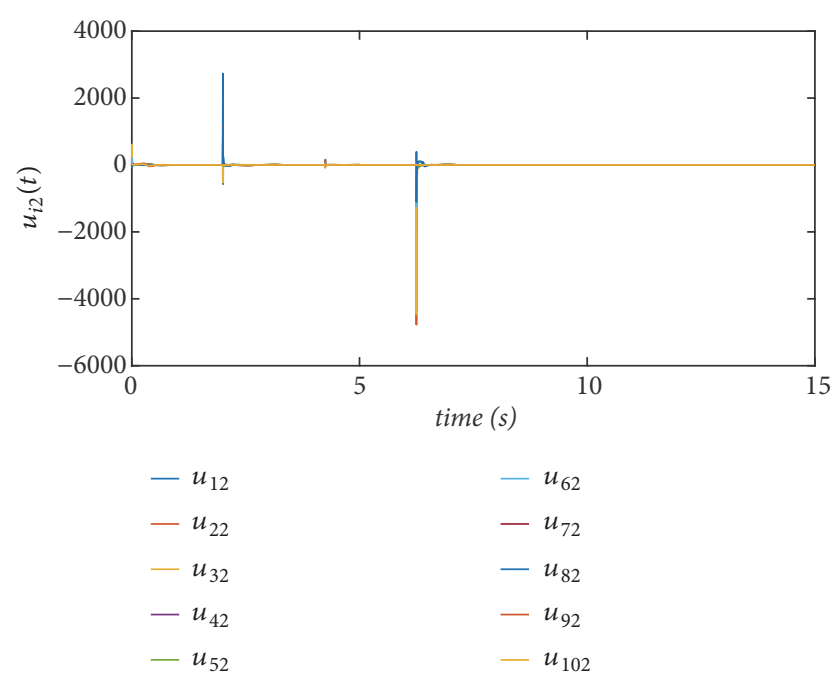

Figure 6: The second component of the controller for each node. 


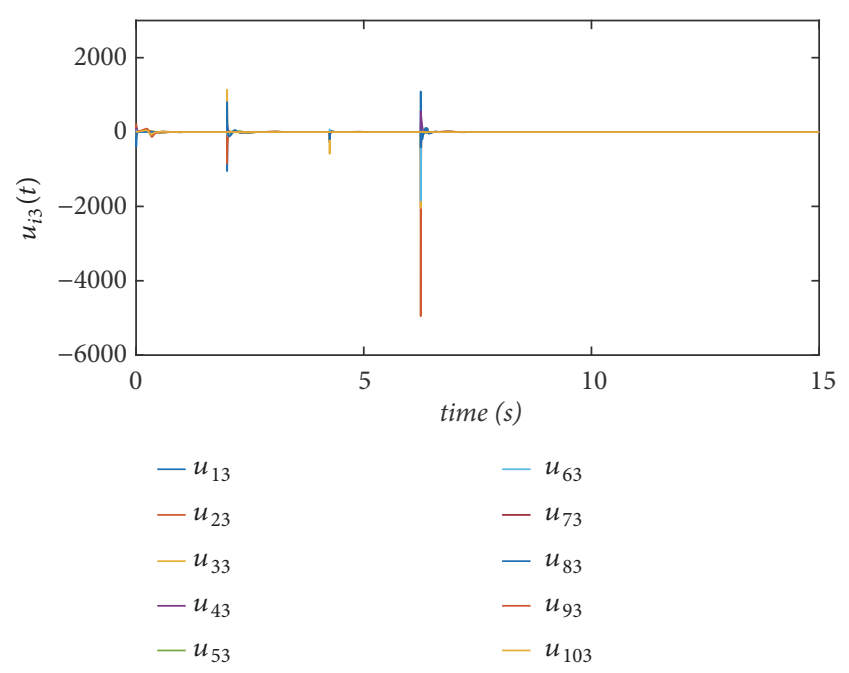

FIgURE 7: The third component of the controller for each node.

Figures 2-4 display the dynamical evolution curves of the synchronization errors $e_{i}(t), i=1,2, \ldots, 10$; as time goes on, the component of errors for each node tends to zero quickly. The aperiodically intermittent controllers are presented in Figures 5-7, from which we can see that all components of the controller are changed violently until they reach zero, which is consistent with the errors evolution results. Moreover, Figure 8 shows the feedback gain convergence to the fixed values: $k_{1}=53.54, k_{2}=5.45, k_{3}=29.01, k_{4}=$ $63.51, k_{5}=8.48, k_{6}=11.97, k_{7}=11.50, k_{8}=2.08, k_{9}=$ $55.79, k_{10}=43.52$. The estimation of unknown periodical coupling strengths is presented in Figure 9. By simulation, we can see that the controlled complex dynamical network (37) achieved exponential synchronization with the help of controller designed in (13) and (14), and the unknown periodical coupling strengths are all estimated successfully.

\section{Conclusions}

In this paper, we investigated aperiodically intermittent synchronization problem of complex dynamical network, which contains unknown periodically coupling strengths and bounded time varying delay and is correspondence with the practical complex network system in a great extent. Based on theories of intermittent control, adaptive control, and learning control, some useful aperiodically intermittent synchronization criteria for complex dynamical network with unknown periodical couplings have been obtained.

Also an illustrative example by the numerical simulation is provided to demonstrate the effectiveness and feasibility of the proposed synchronization method. From the simulation results, we can see that the complex dynamical network is exponentially synchronized when the aperiodically intermittent control is injected, and the coupling strengths is estimated successfully. Meanwhile, in the future, the author will take switching and noise disturbance into account, to study the systems' finite-time aperiodically intermittent synchronization.

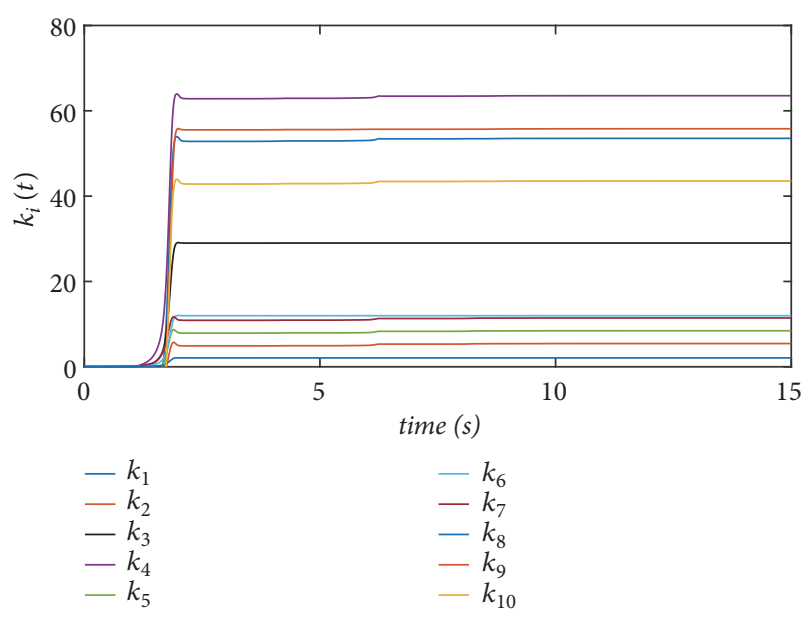

FIGURE 8: The error evolution of the first node.

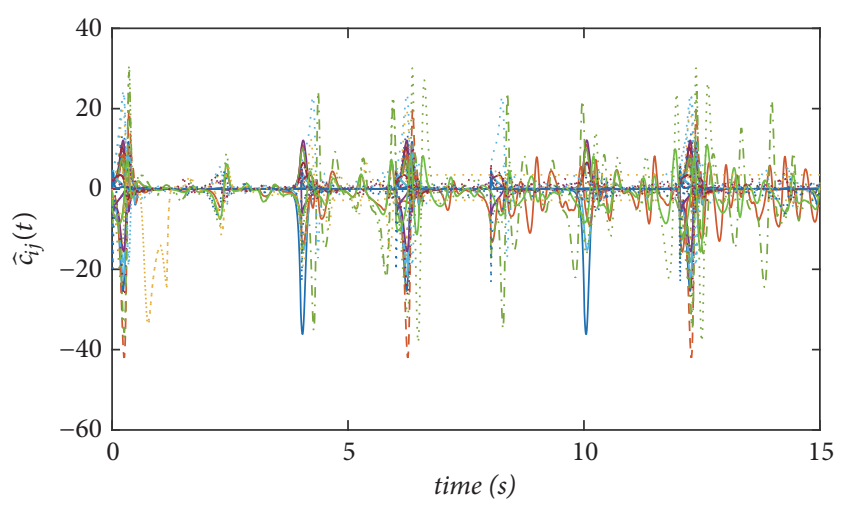

FIGURE 9: The error evolution of the first node.

\section{Data Availability}

The Matlab based models used to support the findings of this study are available from the corresponding author upon request.

\section{Conflicts of Interest}

The authors declare that they have no conflicts of interest.

\section{Acknowledgments}

The work is supported by the National Natural Science Foundation of China under Grant nos. 61573013 and 11705122, the Natural Science Funds of Science and Technology Department of Shaanxi Province under Grant no. 2014JM2-1002, the nature scientific research project of Shaanxi Education Department under Grant no. 18JK0829, and Natural Science Foundation of Xianyang Normal University under Grant no. 14XSYK004.

\section{References}

[1] J.-L. Wang, H.-N. Wu, and T. Huang, Analysis and Control of Output Synchronization for Complex Dynamical Networks, Springer, 2019. 
[2] P. He, "Pinning control and adaptive control for synchronization of linearly coupled reaction-diffusion neural networks with mixed delays," International Journal of Adaptive Control and Signal Processing, vol. 32, no. 8, pp. 1103-1123, 2018.

[3] G. He, J.-a. Fang, and Z. Li, "Finite-time synchronization of cyclic switched complex networks under feedback control," Journal of The Franklin Institute, vol. 354, no. 9, pp. 3780-3796, 2017.

[4] A. Ghaffari and S. Arebi, "Pinning control for synchronization of nonlinear complex dynamical network with suboptimal SDRE controllers," Nonlinear Dynamics, vol. 83, no. 1-2, pp. 1003-1013, 2016.

[5] X. Guo and J. Li, "A new synchronization algorithm for delayed complex dynamical networks via adaptive control approach," Communications in Nonlinear Science and Numerical Simulation, vol. 17, no. 11, pp. 4395-4403, 2012.

[6] P. Selvaraj, O. Kwon, and R. Sakthivel, "Disturbance and uncertainty rejection performance for fractional-order complex dynamical networks," Neural Networks, vol. 112, pp. 73-84, 2019.

[7] P. Selvaraj, R. Sakthivel, and C. K. Ahn, "Observer-based synchronization of complex dynamical networks under actuator saturation and probabilistic faults," IEEE Transactions on Systems, Man, and Cybernetics: Systems, vol. 99, pp. 2168-2216, 2018.

[8] H. Dai, J. Jia, L. Yan, F. Wang, and W. Chen, "Event-triggered exponential synchronization of complex dynamical networks with cooperatively directed spanning tree topology," Neurocomputing, vol. 330, pp. 355-368, 2019.

[9] J. Mei, M. Jiang, Z. Wu, and X. Wang, "Periodically intermittent controlling for finite-time synchronization of complex dynamical networks," Nonlinear Dynamics, vol. 79, no. 1, pp. 295-305, 2015.

[10] J. Sun, Y. Wu, G. Cui, and Y. Wang, "Finite-time real combination synchronization of three complex-variable chaotic systems with unknown parameters via sliding mode control," Nonlinear Dynamics, vol. 88, no. 3, pp. 1677-1690, 2017.

[11] T. Jing, F. Chen, and X. Zhang, "Finite-time lag synchronization of time-varying delayed complex networks via periodically intermittent control and sliding mode control," Neurocomputing, vol. 199, pp. 178-184, 2016.

[12] M. Zochowski, "Intermittent dynamical control," Physica D: Nonlinear Phenomena, vol. 145, no. 3-4, pp. 181-190, 2000.

[13] J. Mei, M. Jiang, X. Wang, J. Han, and S. Wang, "Finitetime synchronization of drive-response systems via periodically intermittent adaptive control," Journal of The Franklin Institute, vol. 351, no. 5, pp. 2691-2710, 2014.

[14] M. Zheng, L. Li, H. Peng et al., "Finite-time synchronization of complex dynamical networks with multi-links via intermittent controls," The European Physical Journal B, vol. 89, pp. 1-12, 2016.

[15] Y. Fan, H. Liu, Y. Zhu, and J. Mei, "Fast synchronization of complex dynamical networks with time-varying delay via periodically intermittent control," Neurocomputing, vol. 205, pp. 182-194, 2016.

[16] L. Li, Z. Tu, J. Mei, and J. Jian, "Finite-time synchronization of complex delayed networks via intermittent control with multiple switched periods," Nonlinear Dynamics, vol. 85, no. 1 , pp. 375-388, 2016.

[17] H. Zhao and G. Cai, "Exponential synchronization of complex delayed dynamical networks with uncertain parameters via intermittent control," Advances in Neural Networks, vol. 9377, pp. 91-98, 2015.
[18] J. Qiu, L. Cheng, X. Chen, J. Lu, and H. He, “Semi-periodically intermittent control for synchronization of switched complex networks: a mode-dependent average dwell time approach," Nonlinear Dynamics, vol. 83, no. 3, pp. 1757-1771, 2016.

[19] M. Liu, Z. Yu, H. Jiang, and C. Hu, "Synchronization of complex networks with coupled and self-feedback delays via aperiodically intermittent strategy," Asian Journal of Control, vol. 19, no. 6, pp. 2062-2075, 2017.

[20] M. Liu, H. Jiang, and C. Hu, "Finite-time synchronization of delayed dynamical networks via aperiodically intermittent control," Journal of The Franklin Institute, vol. 354, no. 13, pp. 5374-5397, 2017.

[21] J. Wang, "Synchronization of delayed complex dynamical network with hybrid-coupling via aperiodically intermittent pinning control," Journal of The Franklin Institute, vol. 354, no. 4, pp. 1833-1855, 2017.

[22] L. Cheng, X. Chen, J. Qiu, J. Lu, and J. Cao, "Aperiodically intermittent control for synchronization of switched complex networks with unstable modes via matrix $\omega$-measure approach," Nonlinear Dynamics, vol. 92, no. 3, pp. 1091-1102, 2018.

[23] W. Jiang, L. Li, Z. Tu, and Y. Feng, "Semiglobal finite-time synchronization of complex networks with stochastic disturbance via intermittent control," International Journal of Robust and Nonlinear Control, vol. 29, no. 8, pp. 2351-2363, 2019.

[24] L. Zhang, X. Yang, C. Xu, and J. Feng, "Exponential synchronization of complex-valued complex networks with timevarying delays and stochastic perturbations via time-delayed impulsive control," Applied Mathematics and Computation, vol. 306, pp. 22-30, 2017.

[25] T. Botmart and P. Niamsup, "Exponential synchronization of complex dynamical network with mixed time-varying and hybrid coupling delays via intermittent control," Advances in Difference Equations, vol. 354, no. 4, pp. 1833-1855, 2014.

[26] M. A. A. Ahmed, Y. Liu, W. Zhang, A. Alsaedi, and T. Hayat, "Exponential synchronization for a class of complex networks of networks with directed topology and time delay," Neurocomputing, vol. 266, pp. 274-283, 2017.

[27] L. Zhang, X. Yang, C. Xu, and J. Feng, "Exponential stability of stochastic differential equations with impulse effects at random times: stochastic differential equations with random impulses," Asian Journal of Control, 2018.

[28] X. Hao and J. Li, "Stochastic synchronization for complex dynamical networks with time-varying couplings," Nonlinear Dynamics, vol. 80, no. 3, pp. 1357-1363, 2015.

[29] P. Selvaraj, R. Sakthivel, and O. M. Kwon, "Finite-time synchronization of stochastic coupled neural networks subject to Markovian switching and input saturation," Neural Networks, vol. 105, pp. 154-165, 2018.

[30] J. Sun, Y. Wang, Y. Wang, and Y. Shen, "Finite-time synchronization between two complex-variable chaotic systems with unknown parameters via nonsingular terminal sliding mode control," Nonlinear Dynamics, vol. 85, no. 2, pp. 1105-1117, 2016.

[31] L. Yan and J. Li, "Adaptive finite-time synchronization for complex dynamical network with different dimensions of nodes and time-varying outer coupling structures," Mathematical Problems in Engineering, vol. 2018, Article ID 2474150, 12 pages, 2018.

[32] X. Liu and T. Chen, "Synchronization of linearly coupled networks with delays via aperiodically intermittent pinning control," IEEE Transactions on Neural Networks and Learning Systems, vol. 26, no. 10, pp. 2396-2407, 2015. 
[33] X. Liu and T. Chen, "Synchronization of complex networks via aperiodically intermittent pinning control," Institute of Electrical and Electronics Engineers Transactions on Automatic Control, vol. 60, no. 12, pp. 3316-3321, 2015. 


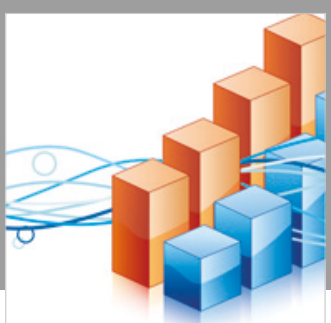

Advances in

Operations Research

\section{-n-m}
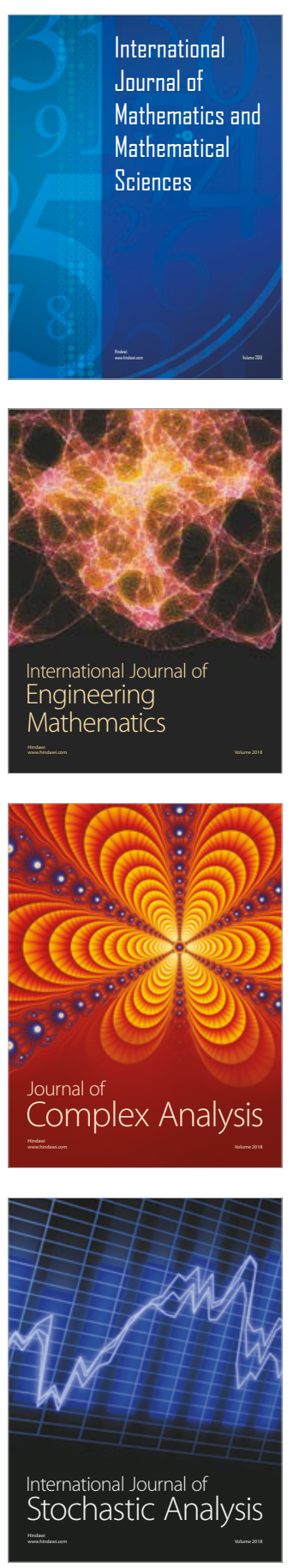
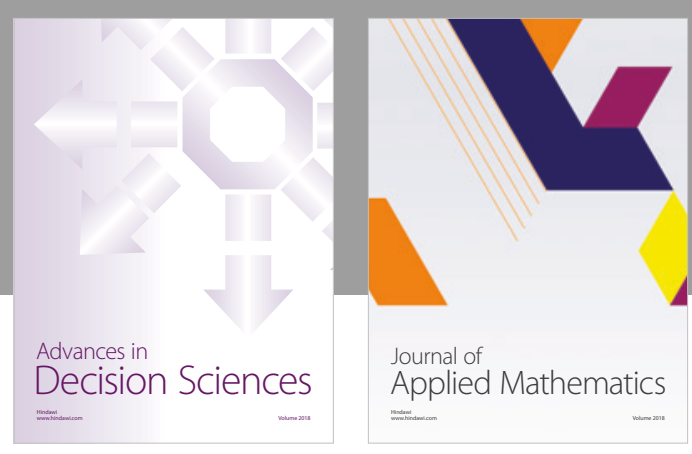

Journal of

Applied Mathematics
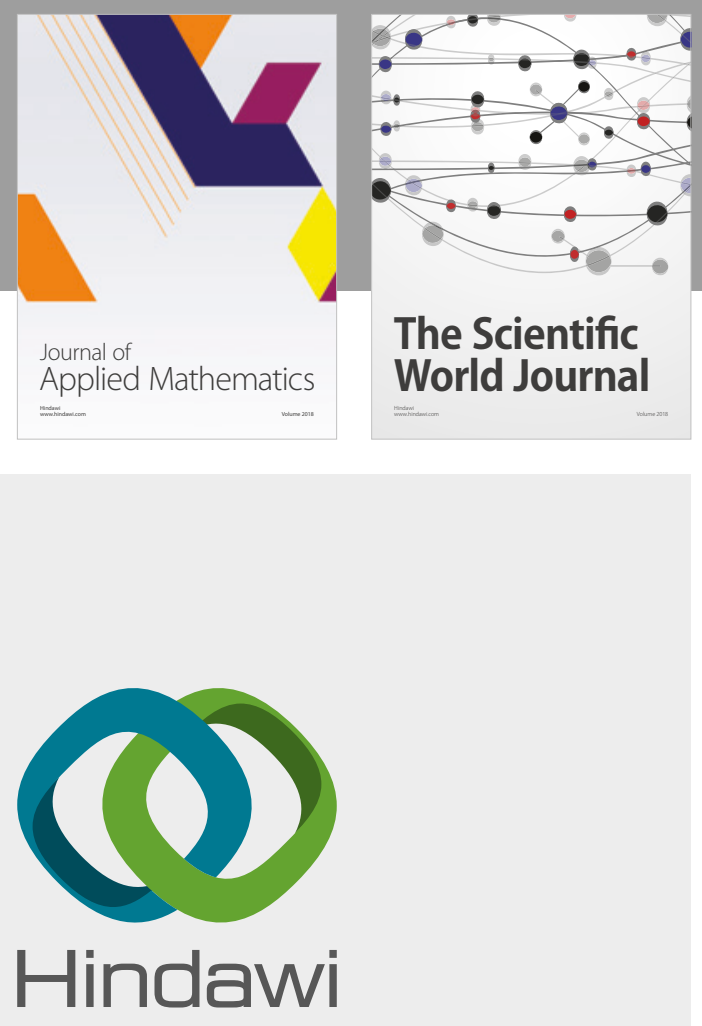

Submit your manuscripts at

www.hindawi.com

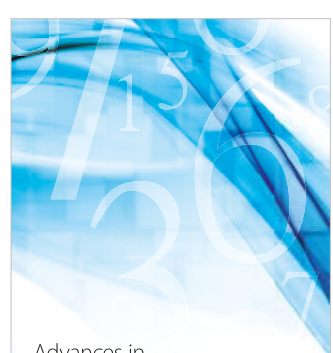

Advances in
Numerical Analysis
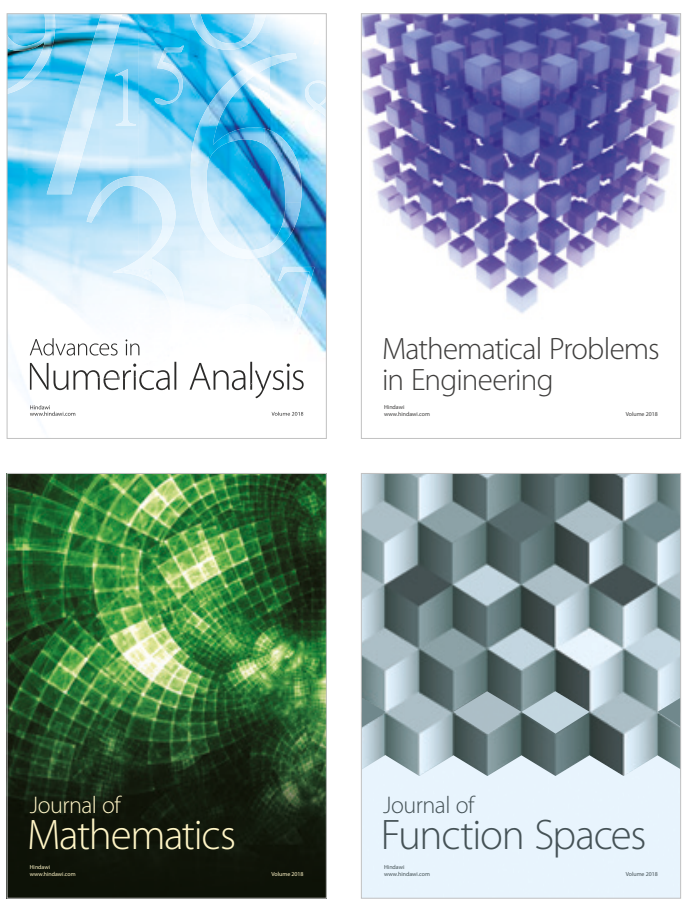

Mathematical Problems in Engineering

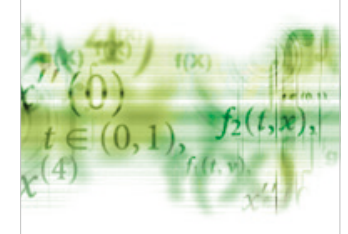

International Journal of

Differential Equations

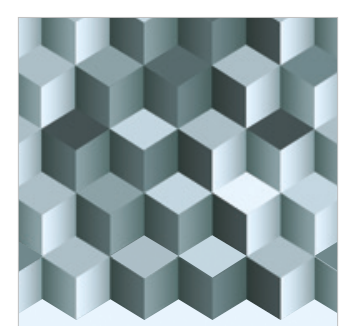

Journal of

Function Spaces

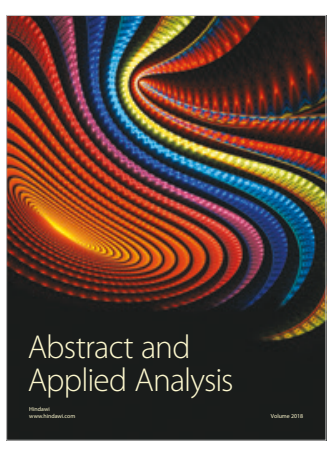

The Scientific

World Journal

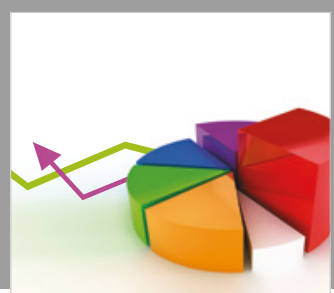

Journal of

Probability and Statistics
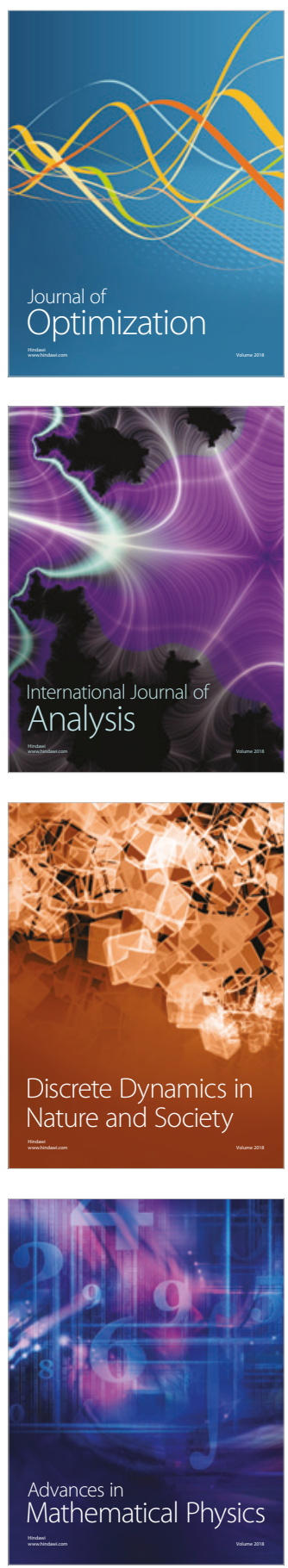